\title{
Compositions of Two Additive Almost Continuous Functions
}

Krzysztof Ciesielski

West Virginia University, krzysztof.ciesielski@mail.wvu.edu

Follow this and additional works at: https://researchrepository.wvu.edu/faculty_publications

Part of the Mathematics Commons

\section{Digital Commons Citation}

Ciesielski, Krzysztof, "Compositions of Two Additive Almost Continuous Functions" (1997). Faculty Scholarship. 831.

https://researchrepository.wvu.edu/faculty_publications/831

This Article is brought to you for free and open access by The Research Repository @ WVU. It has been accepted for inclusion in Faculty Scholarship by an authorized administrator of The Research Repository@ WVU. For more information, please contact ian.harmon@mail.wvu.edu. 
Dariusz Banaszewski, Mathematics Department, Pedagogical University, Chodkiewicza 30, 85-064 Bydgoszcz, Poland.

Krzysztof Ciesielski ; Department of Mathematics, West Virginia University, Morgantown, WV 26506-6310, e-mail: kcies@wvnvms . wvnet.edu.XS

\title{
COMPOSITIONS OF TWO ADDITIVE ALMOST CONTINUOUS FUNCTIONS
}

\begin{abstract}
In the paper we prove that an additive Darboux function $f: \mathbb{R} \rightarrow \mathbb{R}$ can be expressed as a composition of two additive almost continuous (connectivity) functions if and only if either $f$ is almost continuous (connectivity) function or $\operatorname{dim}(\operatorname{ker}(f)) \neq 1$. We also show that for every cardinal number $\lambda \leq 2^{\omega}$ there exists an additive almost continuous functions with $\operatorname{dim}(\operatorname{ker}(f))=\lambda$. A question whether every Darboux function $f: \mathbb{R} \rightarrow \mathbb{R}$ can be expressed as a composition of two almost continuous functions (see [?] or [?]) remains open.
\end{abstract}

\section{Definitions and Notation}

Our terminology and notation is standard. In particular, functions will be identified with their graphs, and for a subset $A$ of $\mathbb{R} \times \mathbb{R}$ (possibly, but not necessarily, a graph of a function) we will write $\operatorname{dom}(A)$ and $\operatorname{rng}(A)$ to denote the $x$-projection (the domain) and the $y$-projection (the range) of $A$, respectively. The cardinality of a set $A$ will be denoted by card $(A)$. Cardinals will be identified with the initial ordinals. The cardinality of the set $\mathbb{R}$ of real numbers, the continuum, will be denoted by $2^{\omega}$.

Throughout the paper we will consider $\mathbb{R}$ as a linear space over the field $\mathbb{Q}$ of rational numbers. A linear basis of this space will be referred to as a Hamel basis. It is evident that the cardinality of every Hamel basis is equal to $2^{\omega}$.

Key Words: Darboux function, connectivity function, almost continuous function, additive function, composition of functions.

Mathematical Reviews subject classification: Primary: 26A15; Secondary: 26A51.

Received by the editors March 6, 1997

* Supported in part by the Polish Academy of Science PAN and by a 1996/97 West Virginia University Senate Research Grant.

${ }^{\dagger}$ Papers authored or co-authored by a Contributing Editor are managed by a Managing Editor or one of the other Contributing Editors. Supported in part by NSF Cooperative Research Grant INT-9600548. 
For an arbitrary set $A \subset \mathbb{R}$ the symbol $L(A)$ will denote the linear subspace of $\mathbb{R}$ over $\mathbb{Q}$ spanned by $A$, i.e., the set of all finite linear combinations of elements of $A$ with coefficients from $\mathbb{Q}$. Similarly for an arbitrary planar set $A \subset \mathbb{R} \times \mathbb{R}$ we define the set $L_{2}(A)$. Also, for $A \subset \mathbb{R}$ and $x \in \mathbb{R}$ we write $x+A$ for $\{x+a: a \in A\}$.

Now, let $L \neq \emptyset$ be a linear subspace of $\mathbb{R}$ over $\mathbb{Q}$. A function $f: L \rightarrow \mathbb{R}$ is said to be additive if it satisfies Cauchy's equation $f(x+y)=f(x)+f(y)$ for every $x, y \in L$. (See [?] or [?, p. 120].) The class of all additive functions from $\mathbb{R}$ to $\mathbb{R}$ will be denoted by $\mathcal{A} d d$. Recall that if $H \subset \mathbb{R}$ is a Hamel basis, then every function $f_{0}: H \rightarrow \mathbb{R}$ can be uniquely extended to the additive function $f: \mathbb{R} \rightarrow \mathbb{R}$. In fact, $f=L_{2}\left(f_{0}\right)$.

For $f \in \mathcal{A} d d$ its kernel $\operatorname{ker}(f)$ is defined as $f^{-1}(0)$. Clearly $\operatorname{ker}(f)$ is a linear subspace of $\mathbb{R}$. Thus, $\operatorname{dim}(\operatorname{ker}(f))$ denotes the (linear) dimension of $\operatorname{ker}(f)$ over $\mathbb{Q}$.

A function $f: \mathbb{R} \longrightarrow \mathbb{R}$ is a Darboux function if it has the intermediate value property, i.e., whenever for every $x_{1}, x_{2} \in \mathbb{R}, x_{1}<x_{2}$, and every point $c$ between $f\left(x_{1}\right)$ and $f\left(x_{2}\right)$ there exists $x \in\left[x_{1}, x_{2}\right]$ such that $f(x)=c$. The family of all Darboux functions from $\mathbb{R}$ to $\mathbb{R}$ will be denoted by $\mathcal{D}$.

A function $f: \mathbb{R} \rightarrow \mathbb{R}$ is said to be almost continuous in the sense of Stallings if each open set (in $\mathbb{R}^{2}$ ) containing $f$ contains also a (graph of) continuous function $g: \mathbb{R} \rightarrow \mathbb{R}[?]$. The class of all almost continuous functions from $\mathbb{R}$ into $\mathbb{R}$ will be denoted by $\mathcal{A C}$.

A closed set $K \subset \mathbb{R} \times \mathbb{R}$ is said to be a blocking set for a function $f: \mathbb{R} \rightarrow \mathbb{R}$ provided $f \cap K=\emptyset$ while $g \cap K \neq \emptyset$ for every continuous function $g: \mathbb{R} \rightarrow \mathbb{R}$. A blocking set $K \subset \mathbb{R} \times \mathbb{R}$ for $f$ is irreducible if no proper subset of $K$ is a blocking set for $f$ [?].

It is known that $f$ is almost continuous if and only if it has no blocking set. Moreover, if $f$ is not almost continuous, then there is an irreducible blocking set $K$ for $f$, and the $x$-projection of $K$ is a non-degenerate connected set [?]. Thus, if $f: \mathbb{R} \rightarrow \mathbb{R}$ intersects all closed sets $K \subset \mathbb{R}^{2}$ with the domain being a non-degenerate interval, then it is almost continuous (cf. [?]).

A function $f: \mathbb{R} \rightarrow \mathbb{R}$ is a connectivity function if its graph is connected (in $\mathbb{R}^{2}$ ). We will use a symbol $\mathcal{C}$ onn to denote the class of all connectivity functions $f: \mathbb{R} \rightarrow \mathbb{R}$. The class of all continuous functions $f: \mathbb{R} \rightarrow \mathbb{R}$ will be denoted by $\mathcal{C}$. We have the following chain of proper inclusions [?].

$$
\mathcal{C} \subset \mathcal{A C} \subset \mathcal{C} \text { onn } \subset \mathcal{D}
$$

It is well-known that the composition of two Darboux functions is a Darboux function again. The problem of characterization of these Darboux functions which can be expressed as a composition of two almost continuous func- 
tions was considered in [?]. (See also [?].) In this paper we will consider the analogous problem in the class of additive functions.

\section{Main Theorem}

Let $\mathcal{B}$ be the family of all closed sets $B \subset \mathbb{R} \times \mathbb{R}$ such that $\operatorname{dom}(B)$ is a non-degenerate interval and either

(A) $B=\mathbb{R} \times\{y\} ;$ or,

(B) $B^{y}=\{x \in \mathbb{R}:\langle x, y\rangle \in B\}$ is nowhere dense for each $y \in \mathbb{R}$.

We will use this family throughout the paper.

In what follows we will use the following lemma repeatedly.

Lemma 1. Let $f \in \mathcal{A} d d$ be such that $\operatorname{ker}(f) \neq\{0\}$. If $f \cap B \neq \emptyset$ for every $B \in \mathcal{B}$, then $f \in \mathcal{A C}$.

Proof. Fix an arbitrary closed set $K \subset \mathbb{R}^{2}$ such that dom $(K)$ is a nondegenerate interval. It is enough to show that $f \cap K \neq \emptyset$. If $K^{y}$ is nowhere dense for each $y \in \mathbb{R}$, then $K \in \mathcal{B}$ and $f \cap K \neq \emptyset$. So, assume otherwise.

Then there is $y \in \mathbb{R}$ such that $K^{y}$ contains a non-degenerate interval $I$. But $\mathbb{R} \times\{y\} \in \mathcal{B} ;$ so $f \cap(\mathbb{R} \times\{y\}) \neq \emptyset$. In particular, there exists $x \in \mathbb{R}$ such that $f(x)=y$. Also, $\operatorname{ker}(f)$ is dense, since $\operatorname{ker}(f) \neq\{0\}$, and so $f^{-1}(y)$ contains a dense set $x+\operatorname{ker}(f)$. Thus $f^{-1}(y) \cap I \supset(x+\operatorname{ker}(f)) \cap I \neq \emptyset$ and $\emptyset \neq f \cap(I \times\{y\}) \subset f \cap K$.

The next theorem constitutes one direction of our main characterization theorem.

Theorem 1. Let $f \in \mathcal{D} \cap \mathcal{A} d d$ be such that $\operatorname{dim}(\operatorname{ker}(f)) \neq 1$. Then $f$ is a composition of two additive almost continuous functions.

Proof. Fix $f \in \mathcal{D} \cap \mathcal{A} d d$ with $\operatorname{dim}(\operatorname{ker}(f)) \neq 1$. If $\operatorname{dim}(\operatorname{ker}(f))=0$, then $f$ is continuous (see [?]) and $f=f \circ i d$. Similarly, if $f \equiv 0$, then $f=f \circ i d$. Hence we can assume that $\operatorname{dim}(\operatorname{ker}(f)) \geq 2$ and $f \not \equiv 0$.

Let $\left\{K_{\alpha}: \alpha<2^{\omega}\right\}$ be an enumeration of the family $\mathcal{B}$ such that $K_{0}=$ $\mathbb{R} \times\{0\}$ and let $\left\{b_{\alpha}: \alpha<2^{\omega}\right\}$ be an enumeration of a fixed Hamel basis with $b_{0} \in \operatorname{ker}(f)$.

We construct, by induction on $\alpha<2^{\omega}$, the sequences $\left\langle g_{\alpha}: \alpha<2^{\omega}\right\rangle$ and $\left\langle h_{\alpha}: \alpha<2^{\omega}\right\rangle$ of additive functions from subsets of $\mathbb{R}$ into $\mathbb{R}$ maintaining the following inductive properties for every $\alpha<2^{\omega}$.

(i) $g_{\beta} \subset g_{\alpha}$ and $h_{\beta} \subset h_{\alpha}$ for every $\beta<\alpha$; 
(ii) $\operatorname{card}\left(\operatorname{dom}\left(g_{\alpha}\right)\right) \leq \max (\omega, \alpha)$, and $\operatorname{card}\left(\operatorname{dom}\left(h_{\alpha}\right)\right) \leq \max (\omega, \alpha)$;

(iii) $\operatorname{rng}\left(g_{\alpha}\right)=\operatorname{dom}\left(h_{\alpha}\right)$ and $h_{\alpha} \circ g_{\alpha}=f \mid \operatorname{dom}\left(g_{\alpha}\right)$;

(iv) $g_{\alpha} \cap K_{\alpha} \neq \emptyset$ and $h_{\alpha} \cap K_{\alpha} \neq \emptyset$;

(v) $b_{\alpha} \in \operatorname{dom}\left(g_{\alpha}\right)$.

To make an inductive step assume that for some $\alpha<2^{\omega}$ the functions $g_{\beta}$ and $h_{\beta}$ satisfying conditions (i)-(v) have already been constructed for every $\beta<\alpha$.

If $\alpha=0$, choose $s_{0} \in \operatorname{ker}(f) \backslash L\left(\left\{b_{0}\right\}\right)$. Such a choice is possible, since $\operatorname{dim}(\operatorname{ker}(f)) \geq 2$. Put $g_{0}=L_{2}\left(\left\{\left\langle b_{0}, 0\right\rangle,\left\langle s_{0}, s_{0}\right\rangle\right\}\right)$ and $h_{0}=L_{2}\left(\left\{\left\langle s_{0}, 0\right\rangle\right\}\right)$. It is easy to see that $g_{0}$ and $h_{0}$ fulfill the conditions $(\mathrm{i})-(\mathrm{v})$.

So, assume that $\alpha>0$ and put $\bar{g}_{\alpha}=\bigcup_{\beta<\alpha} g_{\beta}$ and $\bar{h}_{\alpha}=\bigcup_{\beta<\alpha} h_{\beta}$. Clearly functions $\bar{g}_{\alpha}$ and $\bar{g}_{\alpha}$ satisfy the conditions (i)-(iii). We will find $x_{\alpha}, y_{\alpha}, s_{\alpha}, v_{\alpha}, c_{\alpha} \in \mathbb{R}$ such that

(a) $\left\langle x_{\alpha}, y_{\alpha}\right\rangle \in K_{\alpha}$;

(b) $\left\langle v_{\alpha}, f\left(s_{\alpha}\right)\right\rangle \in K_{\alpha}$;

(c) $g_{\alpha}=L_{2}\left(\bar{g}_{\alpha} \cup\left\{\left\langle x_{\alpha}, y_{\alpha}\right\rangle,\left\langle b_{\alpha}, c_{\alpha}\right\rangle,\left\langle s_{\alpha}, v_{\alpha}\right\rangle\right\}\right)$ and $h_{\alpha}=L_{2}\left(\bar{h}_{\alpha} \cup\left\{\left\langle y_{\alpha}, f\left(x_{\alpha}\right)\right\rangle,\left\langle c_{\alpha}, f\left(b_{\alpha}\right)\right\rangle,\left\langle v_{\alpha}, f\left(s_{\alpha}\right)\right\rangle\right\}\right)$ remain functions.

It is easy to see that such $g_{\alpha}$ and $h_{\alpha}$ will satisfy the conditions (i)-(v).

As a first step we will construct $x_{\alpha}$ and $y_{\alpha}$. If $K_{\alpha} \cap \bar{g}_{\alpha} \neq \emptyset$, we simply choose $\left\langle x_{\alpha}, y_{\alpha}\right\rangle \in K_{\alpha} \cap \bar{g}_{\alpha}$. So, assume that $K_{\alpha} \cap \bar{g}_{\alpha}=\emptyset$. In this case we will find $\left\langle x_{\alpha}, y_{\alpha}\right\rangle \in K_{\alpha}$ such that

$$
x_{\alpha} \notin \operatorname{dom}\left(\bar{g}_{\alpha}\right), \quad \text { and } \quad y_{\alpha} \notin \operatorname{dom}\left(\bar{h}_{\alpha}\right)=\operatorname{rng}\left(\bar{g}_{\alpha}\right) .
$$

Such a restriction is necessary to guarantee condition (c).

Let $X_{\alpha}=\operatorname{dom}\left(\bar{g}_{\alpha}\right)$, and $Y_{\alpha}=\operatorname{dom}\left(\bar{h}_{\alpha}\right)=\operatorname{rng}\left(\bar{g}_{\alpha}\right)$. Then $\operatorname{card}\left(X_{\alpha}\right)<2^{\omega}$ and card $\left(Y_{\alpha}\right)<2^{\omega}$. If $K_{\alpha}$ was chosen according to the part (A) of the definition of $\mathcal{B}$, then $K_{\alpha}=\mathbb{R} \times\{y\}$ for some $y \in \mathbb{R}$. Hence $y \notin Y_{\alpha}$, since $K_{\alpha} \cap \bar{g}_{\alpha}=\emptyset$. Put $y_{\alpha}=y$ and choose $x_{\alpha} \notin X_{\alpha}$. Then $\left\langle x_{\alpha}, y_{\alpha}\right\rangle \in K_{\alpha}$ and the condition (??) is satisfied. So, assume that $K_{\alpha}$ was chosen according to the part (B) of the definition of $\mathcal{B}$, i.e., that $K_{\alpha}^{y}$ is nowhere dense for every $y \in \mathbb{R}$. To deal with this case recall the following fact. (See [?, Th. 29.19, p. 231].)

For every closed set $K \subset \mathbb{R}^{2}$ the set

$$
Z(K)=\left\{y \in \mathbb{R}: K^{y} \text { contains a non-empty perfect set }\right\}
$$

is either countable or is of power continuum. 
This leads us to the two natural subcases.

- $\operatorname{card}\left(Z\left(K_{\alpha}\right)\right)=2^{\omega}$. Then $\operatorname{card}\left(Z\left(K_{\alpha}\right) \backslash Y_{\alpha}\right)=2^{\omega}$ and we can choose $y_{\alpha} \in Z\left(K_{\alpha}\right) \backslash Y_{\alpha}$. Moreover, $\operatorname{card}\left(K_{\alpha}^{y_{\alpha}}\right)=2^{\omega}$, and so we can pick $x_{\alpha} \in K_{\alpha}^{y_{\alpha}} \backslash X_{\alpha}$. Then $\left\langle x_{\alpha}, y_{\alpha}\right\rangle \in K_{\alpha}$ satisfies (??).

- $\operatorname{card}\left(Z\left(K_{\alpha}\right)\right) \leq \omega$. Then the set $E_{\alpha}=\operatorname{dom}\left(K_{\alpha}\right) \backslash \bigcup\left\{K_{\alpha}^{y}: y \in Z\left(K_{\alpha}\right)\right\}$ is a residual subset of the interval dom $\left(K_{\alpha}\right)$ since each set $K_{\alpha}^{y}$ is nowhere dense. In particular, card $\left(E_{\alpha}\right)=2^{\omega}$. Moreover, $K_{\alpha}^{y}$ is countable for every $y \in \mathbb{R} \backslash Z\left(K_{\alpha}\right)$. So the set $E_{\alpha}^{1}=E_{\alpha} \backslash\left(X_{\alpha} \cup \bigcup\left\{K_{\alpha}^{y}: y \in Y_{\alpha} \backslash Z\left(K_{\alpha}\right)\right\}\right)$ has cardinality $2^{\omega}$. Choose $x_{\alpha} \in E_{\alpha}^{1} \subset \operatorname{dom}\left(K_{\alpha}\right) \backslash\left(X_{\alpha} \cup \cup_{y \in Y_{\alpha}} K_{\alpha}^{y}\right)$ and $y_{\alpha} \in \mathbb{R}$ with $\left\langle x_{\alpha}, y_{\alpha}\right\rangle \in K_{\alpha}$. Then $y_{\alpha} \notin Y_{\alpha}$ and (??) is satisfied.

This finishes the construction of $x_{\alpha}$ and $y_{\alpha}$.

To construct $s_{\alpha}$ and $v_{\alpha}$ first note that by (??),

$$
\underline{g}_{\alpha}=L_{2}\left(\bar{g}_{\alpha} \cup\left\{\left\langle x_{\alpha}, y_{\alpha}\right\rangle\right\}\right), \quad \text { and } \quad \underline{h}_{\alpha}=L_{2}\left(\bar{h}_{\alpha} \cup\left\{\left(y_{\alpha}, f\left(x_{\alpha}\right)\right)\right\}\right)
$$

are the additive functions. If $K_{\alpha} \cap \underline{h}_{\alpha} \neq \emptyset$, we choose $\left\langle v_{\alpha}, w_{\alpha}\right\rangle \in K_{\alpha} \cap \underline{h}_{\alpha}$ and take $s_{\alpha}$ such that $\underline{g}_{\alpha}\left(s_{\alpha}\right)=v_{\alpha}$. Such an $s_{\alpha}$ exists since $\operatorname{dom}\left(\underline{h}_{\alpha}\right)=\operatorname{rng}\left(\underline{g}_{\alpha}\right)$. Then $w_{\alpha}=\underline{h}_{\alpha}\left(v_{\alpha}\right)=\underline{h}_{\alpha}\left(g_{\alpha}\left(s_{\alpha}\right)\right)=f\left(s_{\alpha}\right)$, so the condition (b) is satisfied. So, assume that $K_{\alpha} \cap \underline{h}_{\alpha}=\emptyset$. Then, as in the construction of $x_{\alpha}$ and $y_{\alpha}$, we can find $\left\langle v_{\alpha}, w_{\alpha}\right\rangle \in K_{\alpha}$ such that

$$
v_{\alpha} \notin \operatorname{dom}\left(\underline{h}_{\alpha}\right)=\operatorname{rng}\left(\underline{g}_{\alpha}\right), \quad \text { and } \quad w_{\alpha} \notin \operatorname{rng}\left(\underline{h}_{\alpha}\right) .
$$

Now, note that $\operatorname{rng}(f)=\mathbb{R}$, since $f$ is a non-zero additive Darboux function. Choose $s_{\alpha} \in f^{-1}\left(w_{\alpha}\right)$ and notice that $s_{\alpha} \notin \operatorname{dom}\left(\underline{g}_{\alpha}\right)$ since otherwise $w_{\alpha}=f\left(s_{\alpha}\right)=\underline{h}_{\alpha}\left(\underline{g}_{\alpha}\left(s_{\alpha}\right)\right)=\underline{h}_{\alpha}\left(v_{\alpha}\right) \in \operatorname{rng}\left(\underline{h}_{\alpha}\right)$, contradicting (??). Thus, $\left\langle v_{\alpha}, f\left(s_{\alpha}\right)\right\rangle \in K_{\alpha}$, as required in (b).

Finally, to choose $c_{\alpha}$ note that

$$
G_{\alpha}=L_{2}\left(\underline{g}_{\alpha} \cup\left\{\left\langle s_{\alpha}, v_{\alpha}\right\rangle\right\}\right), \quad \text { and } \quad H_{\alpha}=L_{2}\left(\underline{h}_{\alpha} \cup\left\{\left\langle v_{\alpha}, f\left(s_{\alpha}\right)\right\rangle\right\}\right)
$$

are the additive functions. If $b_{\alpha} \in \operatorname{dom}\left(G_{\alpha}\right)$, we put $c_{\alpha}=G_{\alpha}\left(b_{\alpha}\right)$. Otherwise we choose $c_{\alpha} \in \mathbb{R} \backslash \operatorname{dom}\left(H_{\alpha}\right)$. It is easy to see that $x_{\alpha}, y_{\alpha}, s_{\alpha}, v_{\alpha}$, and $c_{\alpha}$ chosen above satisfy (a)-(c). This finishes the inductive construction.

Having constructed functions $g_{\alpha}$ and $h_{\alpha}$ let

$$
g=\bigcup_{\alpha<2^{\omega}} g_{\alpha}, \quad \text { and } \quad h^{0}=\bigcup_{\alpha<2^{\omega}} h_{\alpha} .
$$

It is easy to see that $g$ and $h^{0}$ are additive functions such that $\operatorname{dom}(g)=\mathbb{R}$ (by $(\mathrm{v})$ ) and that $f=h^{0} \circ g$. Now, if $h: \mathbb{R} \rightarrow \mathbb{R}$ is any additive extension of 
$h^{0}$, then, by (iv), $g$ and $h$ are almost continuous, while we still have $f=h \circ g$. This finishes the proof.

Next we will prove the converse of Theorem ??. For this we will need the following simple and well known fact.

Lemma 2. If $g, h \in \mathcal{A} d d$ and $g$ is a surjection, then

$$
\operatorname{dim}(\operatorname{ker}(h \circ g))=\operatorname{dim}(\operatorname{ker}(h))+\operatorname{dim}(\operatorname{ker}(g)) .
$$

Proof. Let $G, H$ be linearly independent sets such that $L(G)=\operatorname{ker}(g)$ and $L(H)=\operatorname{ker}(h)$. For every $w \in H$ choose $s_{w} \in g^{-1}(w)$ and notice that $F=G \cup\left\{s_{w}: w \in H\right\}$ is linearly independent. Indeed, suppose that

$$
x=\sum_{i=1}^{n} q_{i} v_{i}+\sum_{j=1}^{k} p_{j} s_{w_{j}}=0
$$

for some $n, k \in \mathbb{N}, q_{i}, p_{j} \in \mathbb{Q}, v_{i} \in G$, and $w_{j} \in H$, where $i=1, \ldots, n$, and $j=1, \ldots, k$. Then

$$
g(x)=\sum_{i=1}^{n} q_{i} g\left(v_{i}\right)+\sum_{j=1}^{k} p_{j} g\left(s_{w_{j}}\right)=\sum_{j=1}^{k} p_{j} g\left(s_{w_{j}}\right)=\sum_{j=1}^{k} p_{j} w_{j}=0
$$

which shows that $p_{j}=0$ for $j=1, \ldots, k$. Hence, by (??), $\sum_{i=1}^{n} q_{i} v_{i}=0$, which implies that $q_{i}=0$ for $i=1, \ldots, n$.

It is easy to see that $L(F)=\operatorname{ker}(h \circ g)$ and consequently,

$\operatorname{dim}(\operatorname{ker}(g))+\operatorname{dim}(\operatorname{ker}(h))=\operatorname{card}(G)+\operatorname{card}(H)=\operatorname{card}(F)=\operatorname{dim}(\operatorname{ker}(h \circ g))$.

This finishes the proof.

With this lemma in hand we are ready for the next theorem.

Theorem 2. Assume $f \in \mathcal{A} d d$ and $\operatorname{dim}(\operatorname{ker}(f))=1$.

(I) If $f \notin \mathcal{A C}$, then $f=h \circ g$ for $n o h, g \in \mathcal{A} d d \cap \mathcal{A C}$.

(II) If $f \notin \mathcal{C}$ onn, then $f=h \circ g$ for no $h, g \in \mathcal{A} d d \cap \mathcal{C}$ onn.

Proof. Fix $f \in \mathcal{A} d d \cap \mathcal{D}$ such that $\operatorname{dim}(\operatorname{ker}(f))=1$ and suppose that there exist $g, h \in \mathcal{A} d d \cap \mathcal{D}$ with $f=h \circ g$. Then, $g$ is surjection, since $g \not \equiv 0$. By Lemma ??, either $\operatorname{dim}(\operatorname{ker}(g))=0$ or $\operatorname{dim}(\operatorname{ker}(h))=0$. Consequently, either $g$ or $h$ is a Darboux injection, so it is equal to a linear homeomorphism $L(x)=a x$. (Any other additive function has a dense graph, so it cannot be 
Darboux and one-to-one at the same time.) Since the classes $\mathcal{A C}$ and $\mathcal{C}$ onn are closed under composition with homeomorphisms (cf, e.g., [?]), we conclude that $f \in \mathcal{A C}(f \in \mathcal{C}$ onn $)$ if and only if $g, h \in \mathcal{A C}(g, h \in \mathcal{C}$ onn $)$.

Theorems ?? and ?? give us as a corollary the main characterization. (Since $\mathcal{A C} \subset$ Conn.)

Corollary 1. Let $f: \mathbb{R} \rightarrow \mathbb{R}$ be an additive Darboux function. Then

(I) $f$ is a composition of two additive almost continuous functions if and only if either $f$ is almost continuous or $\operatorname{dim}(\operatorname{ker}(f)) \neq 1$;

(II) $f$ is a composition of two additive connectivity functions if and only if either $f$ is a connectivity function or $\operatorname{dim}(\operatorname{ker}(f)) \neq 1$.

\section{Final Remarks}

Although Corollary ?? gives a full characterization of additive Darboux functions which can be expressed as a composition of two additive almost continuous (or connectivity) functions it still does not exclude the possibility that every additive Darboux function can be expressed as a such composition. To conclude this, we need also the following example.

Example 1. There exists a function $f: \mathbb{R} \rightarrow \mathbb{R}$ such that $\operatorname{dim}(\operatorname{ker}(f))=1$ and $f \in \mathcal{A} d d \cap \mathcal{D} \backslash \mathcal{C}$ onn.

Proof. Let $H$ be a Hamel basis and $H_{0}$ be a proper subset of $H$ be with $\operatorname{card}\left(H_{0}\right)=2^{\omega}$. Choose $h_{0} \in H_{0}$, fix a bijection $\varphi: H_{0} \backslash\left\{h_{0}\right\} \longrightarrow H_{0}$ and define $\bar{f}: H \rightarrow \mathbb{R}$ as follows.

$$
\bar{f}(h)=\left\{\begin{array}{lll}
0 & \text { for } & h=h_{0} \\
\varphi(h) & \text { for } & h \in H_{0} \backslash\left\{h_{0}\right\} \\
h & \text { for } & h \in H \backslash H_{0} .
\end{array}\right.
$$

Let $f$ be the additive extension of $\bar{f}$. It is easy to observe that

$$
\bar{f}(h) \in h+L\left(H_{0}\right) \text { for } h \in H
$$

and therefore

$$
f(x) \in x+L\left(H_{0}\right) \text { for every } x \in \mathbb{R} .
$$

It is obvious that $\operatorname{ker}(f)=L\left(\left\{h_{0}\right\}\right)$. Also $\operatorname{rng}(f)=\mathbb{R}$, since $\operatorname{rng}(\bar{f})=H$. Thus $f^{-1}(y) \neq \emptyset$ for every $y \in \mathbb{R}$. Moreover, since all level sets are congruent under translations and $\operatorname{ker}(f)$ is dense [?], $f^{-1}(y)$ is dense for every $y \in \mathbb{R}$. 
Hence, the graph of $f$ is dense in $\mathbb{R}^{2}$ and $f[J]=\mathbb{R}$ for every interval $J \subset \mathbb{R}$. In particular, $f \in \mathcal{D}$. Moreover, by (??),

$$
f \subset \bigcup_{b \in L\left(H_{0}\right)}\{\langle x, x+b\rangle: x \in \mathbb{R}\}
$$

and consequently, the line $y=x+c$ separates the graph of $f$ for every number $c \in \mathbb{R} \backslash L\left(H_{0}\right)$. So, $f$ is not a connectivity function.

Corollary 2. There exists an additive Darboux function $f: \mathbb{R} \rightarrow \mathbb{R}$ such that $f=h \circ g$ for no $f, g \in \mathcal{A} d d \cap \mathcal{C}$ onn.

Our last theorem is a variation of the example above. For its proof we will need one more easy lemma.

Lemma 3. Let $f$ be an additive function and $F=L_{2}(f \cup\{\langle u, v\rangle\})$ where $u \notin \operatorname{dom}(f)$ and $v \notin \operatorname{rng}(f)$. Then $\operatorname{ker}(F)=\operatorname{ker}(f)$.

Proof. Obviously $\operatorname{ker}(f) \subset \operatorname{ker}(F)$. To prove that $\operatorname{ker}(F) \subset \operatorname{ker}(f)$, fix an arbitrary $x \in \operatorname{ker}(F)$. Then

$$
x=q_{0} u+q_{1} w \text { where } q_{0}, q_{1} \in \mathbb{Q} \text { and } w \in \operatorname{dom}(f) .
$$

Since $F(x)=q_{0} v+q_{1} f(w)=0, q_{0} v=-q_{1} f(w)$. Because $v \notin \operatorname{rng}(f), q_{0}=0$ and consequently, $x \in \operatorname{dom}(f)$. Which shows that $x \in \operatorname{ker}(f)$.

Theorem 3. For every cardinal number $\lambda \leq 2^{\omega}$ there exists an additive almost continuous function $f: \mathbb{R} \rightarrow \mathbb{R}$ such that $\operatorname{dim}(\operatorname{ker}(f))=\lambda$.

Proof. Since the function $f \equiv 0$ is almost continuous and $\operatorname{dim}(\operatorname{ker}(f))=2^{\omega}$ for such $f$, we can assume that $\lambda<2^{\omega}$. If $\lambda=0$, then the identity function $i d$ has required properties and so we may also assume that $\lambda>0$.

Now, let $H \subset \mathbb{R}$ be a Hamel basis and $H_{0} \subset H$ be such that card $\left(H_{0}\right)=\lambda$. Also, let $\left\{b_{\alpha}: \alpha<2^{\omega}\right\}=H \backslash H_{0}$ and choose an enumeration $\left\{K_{\alpha}: \alpha<2^{\omega}\right\}$ of the family $\mathcal{B}$ of blocking sets from Lemma ??, with $K_{0}=\mathbb{R} \times\{0\}$. The construction will be a slight modification of that in the proof of Theorem ??

By transfinite induction construct a sequence $\left\langle f_{\alpha}: \alpha<2^{\omega}\right\rangle$ of additive partial functions from $\mathbb{R}$ into $\mathbb{R}$ such the that following inductive conditions are satisfied for every $\alpha<2^{\omega}$.

(i) $f_{\beta} \subset f_{\alpha}$ for every $\beta<\alpha$;

(ii) $f_{\alpha} \cap K_{\alpha} \neq \emptyset$;

(iii) $b_{\alpha} \in \operatorname{dom}\left(f_{\alpha}\right)$ and $\operatorname{card}\left(f_{\alpha}\right) \leq \max (\lambda, \omega, \alpha)$; 
(iv) $\operatorname{ker}\left(f_{\alpha}\right)=L\left(H_{0}\right)$.

We start the induction by putting $f_{0}=L_{2}\left(\left(H_{0} \times\{0\}\right) \cup\left\{\left\langle b_{0}, 1\right\rangle\right\}\right)$. It is obvious that $f_{0}$ fulfills the conditions (i)-(iv).

To make an inductive step, fix $\alpha<2^{\omega}, \alpha>0$, and assume that we have already chosen functions $f_{\beta}$ for $\beta<\alpha$ which satisfy conditions (i)(iv). If $b_{\alpha} \in \operatorname{dom}\left(\bigcup_{\beta<\alpha} f_{\beta}\right)$, we put $\bar{f}_{\alpha}=\bigcup_{\beta<\alpha} f_{\beta}$. Otherwise, by (iii), $\operatorname{card}\left(\operatorname{rng}\left(\bigcup_{\beta<\alpha} f_{\beta}\right)\right)<2^{\omega}$ and we can choose $c_{\alpha} \in \mathbb{R} \backslash \operatorname{rng}\left(\bigcup_{\beta<\alpha} f_{\beta}\right)$. Put

$$
\bar{f}_{\alpha}=L_{2}\left(\left\{\left\langle b_{\alpha}, c_{\alpha}\right\rangle\right\} \cup \bigcup_{\beta<\alpha} f_{\beta}\right) .
$$

Clearly $\bar{f}_{\alpha}$ satisfies (i), (iii) and (iv). Also, if $K_{\alpha} \cap \bar{f}_{\alpha} \neq \emptyset$, then $f_{\alpha}=\bar{f}_{\alpha}$ satisfies (ii) as well and the construction is completed.

So, assume that $K_{\alpha} \cap \bar{f}_{\alpha}=\emptyset$ and let $X_{\alpha}=\operatorname{dom}\left(\bar{f}_{\alpha}\right)$, and $Y_{\alpha}=\operatorname{rng}\left(\bar{f}_{\alpha}\right)$. From (iii) we have that $\operatorname{card}\left(Y_{\alpha}\right) \leq \operatorname{card}\left(X_{\alpha}\right) \leq \max (\omega, \alpha, \lambda)<2^{\omega}$. We will choose $\left\langle x_{\alpha}, y_{\alpha}\right\rangle \in K_{\alpha}$ such that

$$
x_{\alpha} \notin X_{\alpha} \quad \text { and } \quad y_{\alpha} \notin Y_{\alpha}
$$

and define $f_{\alpha}=L_{2}\left(\bar{f}_{\alpha} \cup\left\{\left\langle x_{\alpha}, y_{\alpha}\right\rangle\right\}\right)$. This will finish the construction since, by Lemma ??, $\operatorname{ker}\left(f_{\alpha}\right)=\operatorname{ker}\left(\bar{f}_{\alpha}\right)=L\left(H_{0}\right)$.

Now, if $K_{\alpha}=\mathbb{R} \times\{y\}$ for some $y \in \mathbb{R}$, then $y_{\alpha}=y \notin Y_{\alpha}$, since $K_{\alpha} \cap \bar{f}_{\alpha}=\emptyset$. Choose an arbitrary $x_{\alpha} \in \mathbb{R} \backslash X_{\alpha}$. Then $\left\langle x_{\alpha}, y_{\alpha}\right\rangle$ satisfies (??).

So, assume that $K_{\alpha}=\mathbb{R} \times\{y\}$ for no $y \in \mathbb{R}$. Then $K_{\alpha}^{y}$ is nowhere dense for every $y \in \mathbb{R}$. Since $Z\left(K_{\alpha}\right)=\left\{y \in \mathbb{R}: K_{\alpha}^{y}\right.$ contains non-empty perfect set $\}$ is either countable or has the cardinality of the continuum, we have the following two cases to consider.

- $\operatorname{card}\left(Z\left(K_{\alpha}\right)\right)=2^{\omega}$. Choose $y_{\alpha} \in Z\left(K_{\alpha}\right) \backslash Y_{\alpha}$. Then $\operatorname{card}\left(K_{\alpha}^{y_{\alpha}}\right)=2^{\omega}$ and we may choose $x_{\alpha} \in K_{\alpha}^{y_{\alpha}} \backslash X_{\alpha}$. Clearly $\left\langle x_{\alpha}, y_{\alpha}\right\rangle$ satisfies (??).

- $\operatorname{card}\left(Z\left(K_{\alpha}\right)\right) \leq \omega$. Then the set

$$
E_{\alpha}=\operatorname{dom}\left(K_{\alpha}\right) \backslash \bigcup\left\{K_{\alpha}^{y}: y \in Z\left(K_{\alpha}\right)\right\}
$$

is residual in $\operatorname{dom}\left(K_{\alpha}\right)$ and the set

$$
E_{\alpha}^{1}=E_{\alpha} \backslash\left(X_{\alpha} \cup \bigcup\left\{K_{\alpha}^{y}: y \in Y_{\alpha} \backslash Z\left(K_{\alpha}\right)\right\}\right)
$$

has the cardinality of the continuum. Choose $x_{\alpha} \in E_{\alpha}^{1}$ and $y_{\alpha} \in \mathbb{R}$ such that $\left\langle x_{\alpha}, y_{\alpha}\right\rangle \in K_{\alpha}$. Then $\left\langle x_{\alpha}, y_{\alpha}\right\rangle$ satisfies (??) as well. 
This finishes the inductive construction.

Now, put

$$
f=\bigcup_{\alpha<2^{\omega}} f_{\alpha}
$$

It is easy to see that $f$ has the desired properties.

\section{References}

[1] A. L. Cauchy, Cours d'analyse de l'Ecole Polytechnique, 1. Analyse algébrique, V., Paris, 1821.

[2] D. Gillespie, A property of continuity, Bull. Amer. Math. Soc. 28 (1922), $245-250$.

[3] Z. Grande, A. Maliszewski, T. Natkaniec, Some problems concerning almost continuous functions, Real Analysis Exchange 20(2) (1994-95), 429432 .

[4] A. S. Kechris, Classical Descriptive Set Theory, Springer, New York, 1994.

[5] K. R. Kellum, Sums and limits of almost continuous functions, Colloq. Math. 31 (1974), 125-128.

[6] K. R. Kellum, Almost continuity and connectivity - sometimes it's as easy to prove a stronger result, Real Analysis Exchange 8(1) (1982-83), $244-252$.

[7] M. Kuczma, An introduction to the theory of functional equations and inequalities. Cauchy's equation and Jensen's inequality, PWN WarszawaKraków-Katowice 1985.

[8] T. Natkaniec, Almost Continuity, Real Analysis Exchange 17 (1991-92), $462-520$.

[9] T. Natkaniec, On compositions and products of almost continuous functions, Fund. Math. 139 (1991), 59-74.

[10] J. Stallings, Fixed point theorem for connectivity maps, Fund. Math. 47 (1959), 249-263. 